\title{
Asymptotic expansions of oscillatory integrals with complex phase
}

\author{
Robin Pemantle and Mark C. Wilson
}

\begin{abstract}
We consider saddle point integrals in $d$ variables whose phase functions are neither real nor purely imaginary. Results analogous to those for Laplace (real phase) and Fourier (imaginary phase) integrals hold whenever the phase function is analytic and nondegenerate. These results generalize what is well known for integrals of Laplace and Fourier type. The proofs are via contour shifting in complex $d$-space. This work is motivated by applications to asymptotic enumeration.
\end{abstract}

\section{Introduction}

Integrals of the form

$$
\mathcal{J}(\lambda):=\mathcal{J}(\lambda ; \phi, A):=\int e^{-\lambda \phi(x)} A(x) \mathrm{d} x
$$

arise in many areas of mathematics. There are many variations. This integral may involve one or more variables; the variables may be real or complex; the integral may be global or taken over a small neighborhood or oddly shaped set; varying degrees of smoothness may be assumed; and varying degrees of degeneracy may be allowed near the critical points of the phase function, $\phi$. Often what is sought is a leading order estimate of $\mathcal{J}(\lambda)$ as the positive real parameter $\lambda$ tends to $\infty$, or an asymptotic series $\mathcal{J}(\lambda) \sim \sum_{n} c_{n} g_{n}(\lambda)$, where $\left\{g_{n}\right\}$ is a sequence of elementary functions and the expansion is possibly nowhere convergent, but satisfies

$$
\mathcal{J}(\lambda)-\sum_{n=0}^{N-1} c_{n} g_{n}(\lambda)=O\left(g_{N}(\lambda)\right)
$$

for all $N \geqslant 1$.

In recent work on the asymptotics of multivariate generating functions [PW02; PW04; PW08; BBBP08; BP08; BP04], we have required results of this type in the case where the phase function $\phi$ and the amplitude function $A$ are analytic functions of several variables. The phase function is typically neither real nor purely

2000 Mathematics Subject Classification. Primary: 41A60; Secondary: 30E15.

Key words and phrases. Laplace, Fourier, smooth, analytic, contour methods, Cauchy integral formula, saddle point, stationary phase.

Research supported in part by U.S. National Science Foundation grant DMS-0603821. 
imaginary, but satisfies $\operatorname{Re}\{\phi\} \geqslant 0$. The domain of integration in many of these cases is the product of a manifold with a closed simplex. Although results are known that apply to these cases, the state of the literature is quite poor from the standpoint of ready application. We therefore wrote this note to remedy this situation and to supply what would have been ghost citations in [PW04, Lemmas 4.7 and 4.8].

In Section 7, we review the existing literature in greater detail. To explain briefly the relation between the present results and previous work, we first discuss two special cases in which the results are well known, but by substantially different methods.

Integrals of Fourier type. Let $f, A: \mathbb{R}^{d} \rightarrow \mathbb{R}$ be smooth (that is, $C^{\infty}$ ) functions of $d$ real variables, with $A$ having compact support. Taking $\phi=-\mathrm{i} f$ gives the Fourier-type integral

$$
\mathcal{J}(\lambda)=\int e^{\mathrm{i} \lambda f(x)} A(x) \mathrm{d} x
$$

The standard method of studying this integral is as follows. If $f$ has no critical points in the support of $A$, then integration by parts shows $\mathcal{J}(\lambda)$ to be rapidly decreasing: $\mathcal{J}(\lambda)=O\left(\lambda^{-N}\right)$ for all positive integers, $N$. Using a partition of unity, the integral may therefore be localized to neighborhoods of the critical points of $f$. At an isolated critical point $\nabla f$ vanishes; if the Hessian matrix is non-degenerate then the Morse lemma produces a smooth change of variables under which $f(x)=$ $S_{ \pm}(x):=\sum_{j=1}^{d}( \pm)_{j} x_{j}^{2}$. This reduces the general problem to the case $\phi=\mathrm{i} S$. To solve this, expand $A$ in a series. Each term may be explicitly integrated, resulting in an expansion in decreasing powers of $\lambda$ :

$$
\mathcal{J}(\lambda)=\sum_{n \geqslant 0} c_{n} \lambda^{-d / 2-n}
$$

To see that the resulting series for $\mathcal{J}(\lambda)$ satisfies (1.2), one uses integration by parts again to bound the remainder term. The coefficients $\left\{c_{n}\right\}$ are determined by the derivatives of $A$ and $\phi$. A particularly lucid treatment of this may be found in the first two sections of [Ste93, Chapter VIII].

Integrals of Laplace type. The other well studied case is the Laplace-type integral, where $\phi$ is real. Localization of the integral to the minima of $\phi$ is immediate because the integrand is exponentially small elsewhere. Integrating over balls whose radius has order $\lambda^{-1 / 2}$ shows that near a quadratically nondegenerate minimum, $x_{0}$, the bound $A(x)=O\left(\left|x-x_{0}\right|^{N}\right)$ translates into the bound

$$
\mathcal{J}(\lambda)=O\left(\lambda^{-(d+N) / 2}\right) .
$$

Again one changes variables, expands $A$ into a power series, and integrates term by term, to obtain the series

$$
\mathcal{J}(\lambda)=\sum_{n \geqslant 0} c_{n} \lambda^{-(d+n) / 2} .
$$

Applying (1.4) to the integral of the remainder term shows that (1.5) is an asymptotic expansion for $\mathcal{J}(\lambda)$. Classical treatments of integrals of Laplace type may be found in many places [BH86; Won89], often accompanied by separate treatments of the Fourier case. 
Complex methods. The series (1.3) and (1.5) are formally identical. Further inspection of formulae for $c_{n}$ in the literature shows these to be nearly the same as well, differing only by constant factors of unit modulus. This points to the possibility of unifying the results and generalizing to arbitrary complex functions. An assumption of analyticity is required; technically, this is stronger than smoothness but in practice one is never satisfied without the other. Assuming analyticity, derivations in the Fourier and Laplace cases may indeed be unified via a hybrid approach. In one variable, this is carried out all the time under various names such as "steepest descent," "stationary phase" or "saddle point." If one writes $\mathcal{J}(\lambda)$ as a complex contour integral, the critical point will be a saddle point in $\mathbb{C}^{1}$ for the real part of the phase function; the contour may then be re-oriented to pass through the saddle in the direction of steepest descent of $-\phi$, converting the integral into one of Fourier type and explaining why the series are nearly identical. This is carried out, for example, in [dB81] or [BH86, Chapter 7]. Our aim here is to use similar methods in $d$ dimensions to derive asymptotic expansions for integrals of the form (1.1) such as arise in generating function applications.

The organization of the rest of the paper is as follows. In the next section we give some definitions having to do with stratified spaces. We then state our main result, Theorem 2.3. Section 3 records some easy computations in the case where $\phi$ is the standard phase function $S(\mathbf{x}):=\sum_{j=1}^{d} x_{j}^{2}$. Section 4 handles the general case under the assumption that the real part of $\phi$ has a strict minimum at the critical point. Theorem 2.3 is proved in Section 5. Section 6 gives an application from [PW04] to the estimation of coefficients of a bivariate generating function. Finally, Section 7 discusses the relation to existing literature and further research directions.

\section{Notation and statement of results}

Stratified spaces. Because of the useful properties ensuing from the definition, we shall use Whitney stratified spaces as our chains of integration. Aside from these useful properties, the details of the definition need not concern us, though for completeness we give a precise definition. Let $I$ be a finite partially ordered set and define an $I$-decomposition of a topological space $Z$ to be a partition of $Z$ into a disjoint union of sets $\left\{S_{\alpha}: \alpha \in I\right\}$ such that

$$
S_{\alpha} \cap \overline{S_{\beta}} \neq \varnothing \Longleftrightarrow S_{\alpha} \subseteq \overline{S_{\beta}} \Longleftrightarrow \alpha \leqslant \beta .
$$

Definition 2.1 (Whitney stratification). Let $Z$ be a closed subset of $\mathbb{R}^{n}$. A Whitney stratification of $Z$ is an $I$-decomposition such that

(i) Each $S_{\alpha}$ is a manifold in $\mathbb{R}^{n}$.

(ii) If $\alpha<\beta$, if the sequences $\left\{x_{i} \in S_{\beta}\right\}$ and $\left\{y_{i} \in S_{\alpha}\right\}$ both converge to $y \in S_{\alpha}$, if the lines $l_{i}=\overline{x_{i} y_{i}}$ converge to a line $l$ and the tangent planes $T_{x_{i}}\left(S_{\beta}\right)$ converge to a plane $T$ of some dimension, then both $l$ and $T_{y}\left(S_{\alpha}\right)$ are contained in $T$.

For example, any manifold is a Whitney stratified space with one stratum; any manifold with boundary is a Whitney stratified space with two strata, one being the interior and one the boundary; a $k$-simplex is a Whitney stratified space whose strata are all its faces. Whitney stratified spaces are closed under products and the set of products of strata will stratify the product. Every algebraic variety admits a Whitney stratification, although the singular locus filtration may be too coarse to be a Whitney stratification. We remark that our definition supposes a stratified 
space always to be embedded in $\mathbb{R}^{n}$, but even the more general definition in [GM88] supposes that the relation between the strata is inherited via embedding in some ambient manifold.

To fix ideas, consider the example of a cylinder $S^{1} \times[0,1]$, or more generally, the product of a compact manifold (without boundary) and a simplex. These are precisely the types of domain encountered in applications to asymptotic extraction of coefficients.

Critical points. Associated with the definition of a stratification is the stratified notion of a critical point. Observe that under this definition, any zerodimensional stratum of $\mathcal{M}$ is a critical point of $\mathcal{M}$.

DEFInITIOn 2.2 (smooth functions and their critical points). Say that a function $\phi: \mathcal{M} \rightarrow \mathbb{C}$ on a stratified space $\mathcal{M}$ is smooth if it is smooth when restricted to each stratum. A point $p \in \mathcal{M}$ is said to be critical for the smooth function $\phi$ if and only if the restriction $\mathrm{d} \phi_{\mid S}$ vanishes, where $S$ is the stratum containing $p$.

Let $\mathcal{M} \subseteq \mathbb{C}^{d}$ be a real analytic, $d$-dimensional stratified space. This means that each stratum $S$ is a subset of $\mathbb{C}^{d}$ and each of the chart maps $\psi$ from a neighborhood of the origin in $\mathbb{R}^{k}$ to some $k$-dimensional stratum $S \subseteq \mathbb{C}^{d}$ is analytic (the coordinate functions are convergent power series) with a nonsingular differential. It follows that $\psi$ may be extended to a holomorphic map on a neighborhood of the origin in $\mathbb{C}^{k}$, whose range we denote by $S \otimes \mathbb{C}$. Choosing a small enough neighborhood, we may arrange for $S \otimes \mathbb{C}$ to be a complex $k$-manifold embedded in $\mathbb{C}^{d}$. We say a function $f: \mathcal{M} \rightarrow \mathbb{C}$ is analytic if it has a convergent power series expansion in a neighborhood of every point; an analytic function on $\mathcal{M}$ may be extended to a complex analytic one on a neighborhood of $\mathcal{M}$ in $\mathcal{M} \otimes \mathbb{C}:=\bigcup_{\alpha \in I} S_{\alpha} \otimes \mathbb{C}$. Because we are interested in the integrals of $d$-forms over $\mathcal{M}$, there is no loss of generality in assuming that $\mathcal{M}$ is contained in the closure of its $d$-dimensional strata, whence $\mathcal{M} \otimes \mathbb{C}$ is a neighborhood of $\mathcal{M}$ in $\mathbb{C}^{d}$. Real $d$-manifolds in $\mathbb{C}^{d}$ are not naturally oriented, so we must assume that an orientation is given for each $d$-stratum of $\mathcal{M}$, meaning that the chart maps from $\mathbb{R}^{d}$ to $\mathcal{M}$ must preserve orientations.

Definition. The critical point $p \in \mathcal{M}$ is said to be quadratically nondegenerate if $p$ is in a $d$-dimensional stratum and $\mathcal{H}(p)$ is nonsingular, where

$$
\mathcal{H}(p):=\left(\frac{\partial^{2} \phi}{\partial x_{i} \partial x_{j}}\right)_{1 \leqslant i, j \leqslant d}
$$

is the Hessian matrix for the function $\phi$ of Definition 2.2 on a neighborhood of $p$ in $\mathbb{C}^{d}$

A point $p \in \mathcal{M}$ is said to be stationary if it is critical and $\operatorname{Re}\{\phi(p)\}=0$.

Results. The main result of the paper is an asymptotic expansion for $\mathcal{J}(\lambda)$ in the case where all critical points are in top-dimensional strata and are quadratically nondegenerate. It may seem surprising, after taking the trouble to work in the generality of stratified spaces, to restrict our results to the simplest case. Indeed, combinatorial applications [BM93; PW04; BP04] are known for which the relevant integrals have quadratically nondegenerate critical points in lower-dimensional strata. However, the method in [PW04] is to resolve the multiple intersection via a lifting to a simplex, thereby lifting the integral to an integral over the product of a smooth space with a simplex, with the critical points all lifting to top-dimensional 
strata. In other words, the generality of a stratified domain of integration is needed, but the critical points are forced into top-dimensional strata. We remark that the taxonomy of degeneracies and lower-dimensional critical points is complicated but reasonably well understood [Var77; AGZV88; Wat01].

THEOREM 2.3. Let $\mathcal{M}$ be a compact, real analytic stratified space of dimension $d$ embedded in $\mathbb{C}^{d}$. Let $A$ and $\phi$ be analytic functions on a neighborhood of $\mathcal{M}$ and suppose $\operatorname{Re}\{\phi\} \geqslant 0$ on $\mathcal{M}$. Let $G$ be the subset of stationary points of $\phi$ on $\mathcal{M}$ and assume that $G$ is finite and each stationary point is quadratically nondegenerate. Then the integral $\mathcal{J}(\lambda):=\int_{\mathcal{M}} e^{-\lambda \phi(x)} A(x) \mathrm{d} x$ has an asymptotic expansion

$$
\mathcal{J}(\lambda) \sim \sum_{\ell=0}^{\infty} c_{\ell} \lambda^{-(d+\ell) / 2} .
$$

If $A$ is nonzero at some point of $G$ then the leading term is given by

$$
c_{0}=\pi^{d / 2} \sum_{x \in G} A(x) \omega(x, \lambda)(\operatorname{det} \mathcal{H}(x))^{-1 / 2} .
$$

where $\omega(x, \lambda)$ is the unit complex number $e^{-\lambda \phi(x)}$. The choice of sign of the square root on the right-hand side of (2.1) is determined by choosing any analytic chart map $\psi$ for a neighborhood of $x$ and defining

$$
(\operatorname{det} \mathcal{H}(x))^{-1 / 2}:=(\operatorname{det} \mathcal{H}(\phi \circ \psi))^{-1 / 2} \operatorname{det} J_{\psi},
$$

where $J_{\psi}$ is the Jacobian matrix for $\psi$ at $x$ and the $-1 / 2$ power of the determinant on the right is the product of the inverses of principal square roots of the eigenvalues.

REmark. As usual, by $\int_{\mathcal{M}}$ we mean $\int_{\mathcal{C}}$ for some chain $\mathcal{C}$ representing $\mathcal{M}$.

As mentioned above, analyses involving critical points in lower-dimensional strata are not required. There is one exception, which arises in [PW04, Lemma 4.7 (ii)]. Here, there is a critical point $p \in \partial S$, where $S$ is a top-dimensional stratum, a neighborhood of $p$ in $\bar{S}$ is diffeomorphic to a half-space, and the gradient of $\phi$ at $p$ vanishes not only on the $(d-1)$-dimensional stratum containing $p$ but in the $d$-dimensional half-space neighborhood; quadratic nondegeneracy is assumed in all $d$ dimensions as well.

Corollary 2.4. Assume the hypotheses of Theorem 2.3 except that some of the points $p \in G$ are as in the previous paragraph: critical and nondegenerate in a d-dimensional half-space neighborhood. Then the same conclusion holds, except that the summand in (2.1) corresponding to such a point $p$ must be multiplied by $1 / 2$.

ExAmple 2.5. Let $\mathcal{M}=[0,1] \times(\mathbb{R} / 2 \pi)$ and let $\phi(x, y)=x^{2}+2 \mathrm{i} x \cos (y)$. There are no critical points in the interior. Every point with $x=0$ is a critical point of the stratum $\{0\} \times S^{1}$, but for $(0, y)$ to be a critical point in the half space, the gradient $(2 \mathrm{i} \cos y, 0)$ must vanish, so $y= \pm \pi / 2$. Half the Hessian is

$$
\left[\begin{array}{ll}
1 & \mathrm{i} \\
\mathrm{i} & 0
\end{array}\right]
$$

which is nonsingular. Therefore there are two isolated, quadratically nondegenerate critical points, to which Corollary 2.4 may be applied. Note that the vanishing of $\phi$ on a part of $\partial \mathcal{M}$ renders many classical results inapplicable, though the specific result may be obtained by hand in a variety of ways. 


\section{Preliminary results for the standard phase function}

For $\mathbf{x} \in \mathbb{R}^{d}$, let $S(\mathbf{x}):=\sum_{j=1}^{d} x_{j}^{2}$ denote the standard quadratic form. We begin with a couple of results on integrals of Laplace type where the phase function is the standard quadratic and $A$ is a monomial. In one dimension,

Proposition 3.1.

$$
\int_{-\infty}^{\infty} x^{n} e^{-\lambda x^{2}} \mathrm{~d} x=\frac{n ! \sqrt{\pi}}{(n / 2) ! 2^{n}} \lambda^{-1 / 2-n / 2}
$$

if $n$ is even, while the integral is zero if $n$ is odd.

Proof. For odd $n$ the result is obvious from the fact that the integrand is an odd function. For $n=0$ the result is just the standard Gaussian integral. By induction, assume now the result for $n-2$. Integrating by parts to get the second line, we have

$$
\begin{aligned}
\int_{-\infty}^{\infty} x^{n} e^{-\lambda x^{2}} \mathrm{~d} x & =\int_{-\infty}^{\infty} \frac{-x^{n-1}}{2 \lambda}\left(-2 \lambda x e^{-\lambda x^{2}} \mathrm{~d} x\right) \\
& =\frac{n-1}{2 \lambda} \int_{-\infty}^{\infty} x^{n-2} e^{-\lambda x^{2}} \mathrm{~d} x \\
& =\frac{n-1}{2 \lambda} \frac{(n-2) ! \sqrt{\pi}}{(n / 2-1) ! 2^{n-1}} \lambda^{1 / 2-n / 2}
\end{aligned}
$$

by the induction hypothesis. This is equal to $\lambda^{-1 / 2-n / 2} \sqrt{\pi} n ! /\left((n / 2) ! 2^{n}\right)$, completing the induction.

Corollary 3.2 (monomial integral). Let $\mathbf{r}$ be any d-vector of nonnegative integers and let $\mathbf{x}^{\mathbf{r}}$ denote the monomial $x_{1}^{r_{1}} \cdots x_{d}^{r_{d}}$. Then

$$
\int_{\mathbb{R}^{d}} \mathbf{x}^{\mathbf{r}} e^{-\lambda S(\mathbf{x})} \mathrm{d} \mathbf{x}=\beta_{\mathbf{r}} \lambda^{-(d+|\mathbf{r}|) / 2}
$$

where

$$
\beta_{\mathbf{r}}:=\pi^{d / 2} \prod_{j=1}^{d} \frac{r_{j} !}{\left(r_{j} / 2\right) ! 2^{r_{j}}}
$$

if all the components $r_{j}$ are even, and zero otherwise.

Proof. The integral factors into

$$
\prod_{j=1}^{d}\left[\int_{-\infty}^{\infty} x_{j}^{r_{j}} e^{-\lambda r_{j}^{2}} \mathrm{~d} x_{j}\right] .
$$

To integrate term by term over a Taylor series for $A$, we need the following estimate.

LEMma 3.3 (big-O estimate). Let $A$ be any smooth function satisfying $A(\mathbf{x})=$ $O\left(|\mathbf{x}|^{r}\right)$ at the origin. Then the integral of $A(\mathbf{x}) e^{-\lambda S(\mathbf{x})}$ over any compact set $K$ may be bounded from above as

$$
\int_{K} A(\mathbf{x}) e^{-\lambda S(\mathbf{x})} \mathrm{d} \mathbf{x}=O\left(\lambda^{-(d+r) / 2}\right) .
$$


Proof. Because $K$ is compact and $A(\mathbf{x})=O\left(|\mathbf{x}|^{r}\right)$ at the origin, it follows that there is some constant $c$ for which $|A(\mathbf{x})| \leqslant c|\mathbf{x}|^{r}$ on all of $K$. Let $K_{0}$ denote the intersection of $K$ with the ball $|\mathbf{x}| \leqslant \lambda^{-1 / 2}$, and for $n \geqslant 1$ let $K_{n}$ denote the intersection of $K$ with the shell $2^{n-1} \lambda^{-1 / 2} \leqslant|\mathbf{x}| \leqslant 2^{n} \lambda^{-1 / 2}$. On $K_{0}$ we have

while trivially

$$
|A(\mathbf{x})| \leqslant c \lambda^{-r / 2}
$$

Thus

$$
\int_{K_{0}} e^{-\lambda S(\mathbf{x})} \mathrm{d} \mathbf{x} \leqslant \int_{K_{0}} \mathrm{~d} \mathbf{x} \leqslant c_{d} \lambda^{-d / 2} .
$$

$$
\left|\int_{K_{0}} A(\mathbf{x}) e^{-\lambda S(\mathbf{x})} \mathrm{d} \mathbf{x}\right| \leqslant c^{\prime} \lambda^{-(r+d) / 2} .
$$

For $n \geqslant 1$, on $K \cap K_{n}$, we have the upper bounds

$$
\begin{aligned}
|A(\mathbf{x})| & \leqslant 2^{r n} \quad c \lambda^{-r / 2} \\
e^{-\lambda S(\mathbf{x})} & \leqslant e^{-2^{n-1}} \\
\int_{K_{n}} \mathrm{~d} \mathbf{x} & \leqslant 2^{r n} c_{d} \lambda^{-d / 2} .
\end{aligned}
$$

Letting $c^{\prime \prime}:=c \cdot c_{d} \cdot \sum_{n=1}^{\infty} 2^{2 r n} e^{-2^{n-1}}<\infty$, we may sum to find that

$$
\sum_{n=0}^{\infty}\left|\int_{K_{n}} A(\mathbf{x}) e^{-\lambda S(\mathbf{x})} \mathrm{d} \mathbf{x}\right| \leqslant\left(c^{\prime}+c^{\prime \prime}\right) \lambda^{-(r+d) / 2},
$$

proving the lemma.

It is now easy to compute a series for $\mathcal{J}(\lambda)$ in the case where $\phi$ is the standard quadratic and the integral is over a neighborhood of the origin in $\mathbb{R}^{d}$.

Theorem 3.4 (standard phase). Let $A(\mathbf{x})=\sum_{\mathbf{r}} a_{\mathbf{r}} \mathbf{x}^{\mathbf{r}}$ be an analytic function defined on a neighborhood $\mathcal{N}$ of the origin in $\mathbb{R}^{d}$. Let

$$
\mathcal{J}(\lambda):=\int_{\mathcal{N}} A(\mathbf{x}) e^{-\lambda S(\mathbf{x})} \mathrm{d} \mathbf{x} .
$$

Then

$$
\mathcal{J}(\lambda) \sim \sum_{n}\left[\sum_{|\mathbf{r}|=n} a_{\mathbf{r}} \beta_{\mathbf{r}}\right] \lambda^{-(n+d) / 2}
$$

as an asymptotic series expansion in decreasing powers of $\lambda$, with $\beta_{\mathbf{r}}$ as in (3.1).

Proof. Write $A(\mathbf{x})$ as a power series up to degree $N$ plus a remainder term:

$$
A(\mathbf{x})=\left(\sum_{n=0}^{N} \sum_{|\mathbf{r}|=n} a_{\mathbf{r}} \mathbf{x}^{\mathbf{r}}\right)+R(\mathbf{x})
$$

where $R(\mathbf{x})=O\left(|\mathbf{x}|^{N+1}\right)$. Using Corollary 3.2 to integrate all the monomial terms and Lemma 3.3 to bound the integral of $R(\mathbf{x}) e^{-\lambda S(\mathbf{x})}$ shows that

$$
\mathcal{J}(\lambda)=\sum_{n=0}^{N} \sum_{|\mathbf{r}|=n} a_{\mathbf{r}} \beta_{\mathbf{r}} \lambda^{-(d+n) / 2}+O\left(\lambda^{-(d+n) / 2-1}\right),
$$

which proves the asymptotic expansion. 


\section{The case of a strict minimum}

In this section, we continue to integrate over a neighborhood of the origin in $\mathbb{R}^{d}$, but we generalize to any analytic phase function $\phi$ with the restriction that the real part of $\phi$ have a strict minimum at the origin. The assumption of a strict minimum localizes the integral to the origin, so the only tricky aspects are keeping track of the sign (Lemma 4.3) and being rigorous about moving the contour.

THEOREM 4.1. Let $A$ and $\phi$ be complex-valued analytic functions on a compact neighborhood $\mathcal{N}$ of the origin in $\mathbb{R}^{d}$, and suppose the real part of $\phi$ is nonnegative, vanishing only at the origin. Suppose the Hessian matrix $\mathcal{H}$ of $\phi$ at the origin is nonsingular. Letting $\mathcal{J}(\lambda):=\int_{\mathcal{N}} A(\mathbf{x}) e^{-\lambda \phi(\mathbf{x})} \mathrm{d} \mathbf{x}$, one has an asymptotic expansion

$$
\mathcal{J}(\lambda) \sim \sum_{\ell \geqslant 0} c_{\ell} \lambda^{d / 2-\ell}
$$

where

$$
c_{0}=A(\mathbf{0}) \frac{\pi^{d / 2}}{\sqrt{\operatorname{det} \frac{1}{2} \mathcal{H}}}=A(\mathbf{0}) \frac{(2 \pi)^{d / 2}}{\sqrt{\operatorname{det} \mathcal{H}}}
$$

and the choice of sign is defined by taking the product of the principal square roots of the eigenvalues of $\mathcal{H}$.

The proof is essentially a reduction to the case of standard phase. The key is the well known Morse Lemma. The proof given in [Ste93, VIII:2.3.2] is for the smooth category and for purely real or imaginary phase but extends without significant change to complex values and the analytic category. For completeness, we include the adapted proof.

Lemma 4.2 (Morse lemma). There is a bi-holomorphic change of variables $\mathbf{x}=\psi(\mathbf{y})$ such that $\phi(\psi(\mathbf{y}))=S(\mathbf{y}):=\sum_{j=1}^{d} y_{j}^{2}$. The differential $J_{\psi}=\mathrm{d} \psi(\mathbf{0})$ will satisfy $\left(\operatorname{det} J_{\psi}\right)^{2}=\left(\operatorname{det} \frac{1}{2} \mathcal{H}\right)^{-1}$.

Proof. Addressing the second conclusion first, we recall how the Hessian matrix behaves under a change of variables. If $\psi: \mathbb{C}^{d} \rightarrow \mathbb{C}^{d}$ is bi-holomorphic on a neighborhood of $\mathbf{x}$ and if $\phi$ has vanishing gradient at $\psi(\mathbf{x})$ and Hessian matrix $\mathcal{H}$ there, then the Hessian matrix $\tilde{\mathcal{H}}$ of $\phi \circ \psi$ at $\mathbf{x}$ is given by

$$
\tilde{\mathcal{H}}=J_{\psi}^{T} \mathcal{H} J_{\psi},
$$

where $J_{\psi}$ is the Jacobian matrix for $\psi$ at $\mathbf{x}$. The standard form $S$ has Hessian matrix equal to twice the identity, hence any function $\psi$ satisfying $\phi \circ \psi=S$ must satisfy

$$
2 I=J_{\psi}^{T} \mathcal{H} J_{\psi}
$$

Dividing by two and taking determinants yields $\left|\mathbf{J}_{\psi}\right|^{2} \operatorname{det}\left(\frac{1}{2} \mathcal{H}\right)=1$, proving the second conclusion.

To prove the change of variables, the first step is to write

$$
\phi(\mathbf{x})=\sum_{j, k=1}^{d} x_{j} x_{k} \phi_{j, k},
$$

where the functions $\phi_{j, k}=\phi_{k, j}$ are analytic and satisfy $\phi_{j, k}(\mathbf{0})=(1 / 2) \mathcal{H}_{j, k}$. It is obvious from a formal power series viewpoint that this can be done because the summand $x_{j} x_{k} \phi_{j, k}$ can be any power series with coefficients indexed by the 
orthant $\left\{\mathbf{r}: \mathbf{r} \geqslant \mathbf{e}_{j}+\mathbf{e}_{k}\right\}$. These orthants cover $\{\mathbf{r}:|\mathbf{r}| \geqslant 2\}$, so we may obtain any function $\phi$ vanishing to order two; matching coefficients on the terms of order precisely two shows that $\phi_{j, k}(\mathbf{0})=(1 / 2) \mathcal{H}_{j, k}$.

More constructively, we may give a formula for $\phi_{j, k}$. There is plenty of freedom, but a convenient choice is to let $a_{\mathbf{r}}$ denote the coefficient of $\mathbf{x}^{\mathbf{r}}$ in $\phi(\mathbf{x})$ and to take

$$
x_{k} x_{k} \phi_{j, k}(\mathbf{x}):=\sum_{|\mathbf{r}| \geqslant 2} \frac{r_{j}\left(r_{k}-\delta_{j, k}\right)}{|\mathbf{r}|(|\mathbf{r}|-1)} a_{\mathbf{r}} \mathbf{x}^{\mathbf{r}} .
$$

For fixed $\mathbf{r}$, it is easy to check that

$$
\sum_{1 \leqslant j, k \leqslant d} \frac{r_{j}\left(r_{k}-\delta_{j, k}\right)}{|\mathbf{r}|(|\mathbf{r}|-1)}=1
$$

whence $\phi=\sum x_{j} x_{k} \phi_{j, k}$. Alternatively, the following analytic computation from [Ste93] verifies that $\phi=\sum_{j, k} x_{j} x_{k} \phi_{j, k}$. Any function $f$ vanishing at zero satisfies $f(t)=\int_{0}^{1}(1-s) f^{\prime}(s) d s$, as may be seen by integrating by parts (take $g(s)=$ $-(1-s))$. Fix $\mathbf{x}$ and apply this fact with $f(t)=(\mathrm{d} / \mathrm{d} t) \phi(t \mathbf{x})$ to obtain

$$
\phi(\mathbf{x})=\int_{0}^{1} \frac{\mathrm{d}}{\mathrm{d} t} \phi(t \mathbf{x}) \mathrm{d} t=\int_{0}^{1}(1-t) \frac{\mathrm{d}^{2}}{\mathrm{~d} t^{2}} \phi(t \mathbf{x}) \mathrm{d} t .
$$

The multivariate chain rule gives

$$
\frac{d^{2}}{\mathrm{~d} t^{2}} \phi(t \mathbf{x})=\sum_{j, k} x_{j} x_{k} \frac{\partial^{2} \phi}{\partial x_{j} \partial x_{k}}(t \mathbf{x})
$$

plug in $\phi=\sum_{\mathbf{r}} a_{\mathbf{r}} \mathbf{x}^{\mathbf{r}}$ and integrate term by term using $\int_{0}^{1}(1-t) t^{n-2} \mathrm{~d} t=\frac{1}{n(n-1)}$ to see that $\phi=\sum_{j, k} x_{j} x_{k} \phi_{j, k}$.

The second step is an induction. Suppose first that $\phi_{j, j}(\mathbf{0}) \neq 0$ for all $j$. The function $\phi_{1,1}^{-1}$ and a branch of the function $\phi_{1,1}^{1 / 2}$ are analytic in a neighborhood of the origin. Set

$$
y_{1}:=\phi_{1,1}^{1 / 2}\left[x_{1}+\sum_{k>1} \frac{y_{k} \phi_{1, k}}{\phi_{1,1}}\right] .
$$

Expanding, we find that the terms of $y_{1}^{2}$ of total degree at most one in the terms $x_{2}, \ldots, x_{d}$ match those of $\phi$, and therefore

$$
\phi(\mathbf{x})=y_{1}^{2}+\sum_{j, k \geqslant 2} x_{j} x_{k} h_{j, k}
$$

for some analytic functions $h_{j, k}$ satisfying $h_{j, k}(\mathbf{0})=(1 / 2) \mathcal{H}_{j, k}$. Similarly, if

$$
\phi(\mathbf{x})=\sum_{j=1}^{r-1} y_{j}^{2}+\sum_{j, k \geqslant r} x_{j} x_{k} h_{j, k}
$$

then setting

gives

$$
y_{r}:=\phi_{r, r}^{1 / 2}\left[x_{r}+\sum_{k>r} \frac{y_{k} \phi_{r, k}}{\phi_{r, r}}\right]
$$

$$
\phi(\mathbf{x})=\sum_{j=1}^{r} y_{j}^{2}+\sum_{j, k \geqslant r+1} x_{j} x_{k} \tilde{h}_{j, k}
$$


for some analytic functions $\tilde{h}_{j, k}$ still satisfying $h_{j, k}(\mathbf{0})=(1 / 2) \mathcal{H}_{j, k}$. By induction, we arrive at $\phi(\mathbf{x})=\sum_{j=1}^{d} y_{j}^{2}$, finishing the proof of the Morse Lemma in the case where each $\mathcal{H}_{j, j}$ is nonzero.

Finally, if some $\mathcal{H}_{j, k}=0$, because $\mathcal{H}$ is nonsingular we may always find some unitary map $U$ such that the Hessian $U^{T} \mathcal{H} U$ of $\phi \circ U$ has no vanishing diagonal entry. We know there is a $\psi_{0}$ such that $(\phi \circ U) \circ \psi_{0}=S$, and taking $\psi=U \circ \psi_{0}$ finishes the proof in this case.

Proof of Theorem 4.1. The power series allows us to extend $\phi$ to a neighborhood of the origin in $\mathbb{C}^{d}$. Under the change of variables $\psi$ from the previous lemma, we see that

$$
\begin{aligned}
\mathcal{J}(\lambda) & =\int_{\psi^{-1} \mathcal{C}} A \circ \psi(\mathbf{y}) e^{-\lambda S(\mathbf{y})}(\operatorname{det} \mathrm{d} \psi(\mathbf{y})) \mathrm{d} \mathbf{y} \\
& =\int_{\psi^{-1} \mathcal{C}} \tilde{A}(\mathbf{y}) e^{-\lambda S(\mathbf{y})} \mathrm{d} \mathbf{y}
\end{aligned}
$$

for some analytic function $\tilde{A}$, where $\mathcal{C}$ is a neighborhood of the origin in $\mathbb{R}^{n}$. We need to check that we can move the chain $\psi^{-1} \mathcal{C}$ of integration back to the real plane.

Let $h(\mathbf{z}):=\operatorname{Re}\{S(\mathbf{z})\}$. The chain $\mathcal{C}^{\prime}:=\psi^{-1}(\mathcal{C})$ lies in the region $\left\{\mathbf{z} \in \mathbb{C}^{d}\right.$ : $h(\mathbf{z})>0\}$ except when $\mathbf{z}=0$, and in particular, $h \geqslant \epsilon>0$ on $\partial \mathcal{C}^{\prime}$. Let

$$
H(\mathbf{z}, t):=\operatorname{Re}\{\mathbf{z}\}+(1-t) \mathrm{i} \operatorname{Im}\{\mathbf{z}\} .
$$

In other words, $H$ is a homotopy from the identity map to the map $\pi$ projecting out the imaginary part of the vector $\mathbf{z}$. For any chain $\sigma$, the homotopy $H$ induces a chain homotopy, $H(\sigma)$, supported on the image of the support of $\sigma$ under the homotopy $H$ and satisfying

$$
\partial H(\sigma)=\sigma-\pi \sigma+H(\partial \sigma) .
$$

With $\sigma=\mathcal{C}^{\prime}$, observing that $h(H(\mathbf{z}, t)) \geqslant h(\mathbf{z})$, we see there is a $(d+1)$-chain $\mathcal{D}$ with

$$
\partial \mathcal{D}=\mathcal{C}^{\prime}-\pi \mathcal{C}^{\prime}+\mathcal{C}^{\prime \prime}
$$

and $\mathcal{C}^{\prime \prime}$ supported on $\{h>\epsilon\}$. Stokes' Theorem tells us that for any holomorphic $d$-form $\omega$,

$$
\int_{\partial \mathcal{D}} \omega=\int_{\mathcal{D}} \mathrm{d} \omega=0,
$$

and consequently that

$$
\int_{\mathcal{C}^{\prime}} \omega=\int_{\pi \mathcal{C}^{\prime}} \omega+\int_{\mathcal{C}^{\prime \prime}} \omega .
$$

When $\omega=\tilde{A} e^{-\lambda S} \mathrm{~d} \mathbf{y}$, the integral over $\mathcal{C}^{\prime \prime}$ is $O\left(e^{-\lambda \epsilon}\right)$, giving

$$
\mathcal{J}(\lambda)=\int_{\pi \mathcal{C}^{\prime}} \tilde{A}(\mathbf{y}) e^{-\lambda S(\mathbf{y})} \mathrm{d} \mathbf{y}+O\left(e^{-\epsilon \lambda}\right) .
$$

Up to sign, the chain $\pi \mathcal{C}^{\prime \prime}$ is a disk in $\mathbb{R}^{d}$ with the standard orientation plus something supported in $\{h>\epsilon\}$. To see this, note that $\pi$ maps any real $d$-manifold in $\mathbb{C}^{d}$ diffeomorphically to $\mathbb{R}^{d}$ wherever the tangent space is transverse to the imaginary subspace. The tangent space to the support of $\mathrm{C}^{\prime}$ at the origin is transverse to the imaginary subspace because $S \geqslant 0$ on $\mathrm{C}^{\prime}$, whereas the imaginary subspace is precisely the negative $d$-space of the index- $d$ form $S$. The tangent space varies 
continuously, so in a neighborhood of the origin, $\pi$ is a diffeomorphism. Observing that $\tilde{A}(\mathbf{0})=A(\mathbf{0}) \operatorname{det}(\mathrm{d} \psi(\mathbf{0}))=A(\mathbf{0})(\operatorname{det}(1 / 2) \mathcal{H})^{-1 / 2}$ and using Theorem 3.4 finishes the proof, up to the choice of sign of the square root.

The map $\mathrm{d} \pi \circ \mathrm{d} \psi^{-1}(\mathbf{0})$ maps the standard basis of $\mathbb{R}^{d}$ to another basis for $\mathbb{R}^{d}$. Verifying the sign choice is equivalent to showing that this second basis is positively oriented if and only if $\operatorname{det}(\mathrm{d} \psi(\mathbf{0}))$ is the product of the principal square roots of the eigenvalues of $\mathcal{H}$ (it must be either this or its negative). Thus we shall be finished if we apply the following lemma (with $\alpha=\psi^{-1}$ ).

Lemma 4.3. Let $W \subseteq \mathbb{C}^{d}$ be the set $\{\mathbf{z}: \operatorname{Re}\{S(\mathbf{z})\}>0\}$. Pick any $\alpha \in G L_{d}(\mathbb{C})$ mapping $\mathbb{R}^{d}$ into $\bar{W}$, and let $M:=\alpha^{T} \alpha$ be the matrix representing the composition $S \circ \alpha$. Let $\pi: \mathbb{C}^{d} \rightarrow \mathbb{R}^{d}$ be projection onto the real part. Then $\pi \circ \alpha$ is orientation preserving on $\mathbb{R}^{d}$ if and only if $\operatorname{det} \alpha$ is the product of the principal square roots of the eigenvalues of $M$ (rather than the negative of this product).

Proof. First suppose $\alpha \in G L_{d}(\mathbb{R})$. Then $M$ has positive eigenvalues, so the product of their principal square roots is positive. The map $\pi$ is the identity on $\mathbb{R}^{d}$ so the statement boils down to saying that $\alpha$ preserves orientation if and only if it has positive determinant, which is true by definition. In the general case, let $\alpha_{t}:=\pi_{t} \circ \alpha$, where $\pi_{t}(\mathbf{z})=\operatorname{Re}\{\mathbf{z}\}+(1-t) \operatorname{Im}\{\mathbf{z}\}$. As we saw in the previous proof, $\pi_{t}\left(\mathbb{R}^{d}\right) \subseteq \bar{W}$ for all $0 \leqslant t \leqslant 1$, whence $M_{t}:=\alpha_{t}^{T} \alpha_{t}$ has eigenvalues with nonnegative real parts. The product of the principal square roots of the eigenvalues is a continuous function on the set of nonsingular matrices with no negative real eigenvalues. The determinant of $\alpha_{t}$ is a continuous function of $t$, and we have seen that it agrees with the product of principal square roots of eigenvalues of $M_{t}$ when $t=1$ (the real case). So by continuity, this is the correct sign choice for all $0 \leqslant t \leqslant 1$; taking $t=0$ proves the lemma.

For later use, we record one easy corollary of Theorem 4.1.

Corollary 4.4. Assume the hypotheses of Theorem 4.1 and let $\mathcal{N}^{\prime}$ be the intersection of $\mathcal{N}$ with a region diffeomorphic to a half-space through the origin. If $A(\mathbf{0}) \neq 0$ then

$$
\mathcal{J}^{\prime}(\lambda):=\int_{\mathcal{N}^{\prime}} A(\mathbf{x}) e^{-\lambda \phi(\mathbf{x})} \mathrm{d} x \sim \frac{c_{0}}{2} \lambda^{-d / 2},
$$

where $c_{0}$ is the same as in the conclusion of Theorem 4.1.

Proof. Under the change of variables $\psi$ and the projection $\pi$, this region maps to a region $\mathcal{N}^{\prime \prime}$ diffeomorphic to a half-space with the origin on the boundary. Changing variables by $\mathbf{y}=\lambda^{-1 / 2} \mathbf{x}$ and writing $\mathcal{N}_{\lambda}$ for $\lambda^{1 / 2} \mathcal{N}^{\prime \prime}$, we have

$$
\mathfrak{J}^{\prime}(\lambda)=\lambda^{-d / 2} \int_{\mathcal{N}_{\lambda}} A_{\lambda}(\mathbf{y}) e^{-S(\mathbf{y})} \mathrm{d} \mathbf{y},
$$

where $A_{\lambda}(\mathbf{y})=(A \circ \psi)\left(\lambda^{-1 / 2} \mathbf{y}\right)$. The function $A_{\lambda}$ converges to $A(\mathbf{0})$ pointwise and also in $L^{2}(\mu)$, where $\mu$ is the Gaussian measure $e^{-S(\mathbf{x})} \mathrm{d} \mathbf{x}$. Also, the regions $\mathcal{N}_{\lambda}$ converge to a half-space $H$ in the sense that their indicators $\mathbf{1}_{\mathcal{N}_{\lambda}}$ converge to $\mathbf{1}_{H}$ in $L^{2}(\mu)$. Thus $A_{\lambda} \mathbf{1}_{\mathcal{N}_{\lambda}}$ converges to $A(\mathbf{0}) \mathbf{1}_{H}$ in $L^{1}(\mu)$, and unravelling this statement we see that

$$
\int_{\mathcal{N}_{\lambda}} A_{\lambda}(\mathbf{y}) e^{-S(\mathbf{y})} \mathrm{d} \mathbf{y} \rightarrow \int_{H} A(\mathbf{0}) e^{-S(\mathbf{y})} \mathrm{d} \mathbf{y} .
$$


The last quantity is equal to $c_{0} / 2$, showing that $\lambda^{d / 2} \mathcal{J}^{\prime}(\lambda) \rightarrow c_{0} / 2$ and finishing the proof.

\section{Proofs of main results}

Theorem 2.3 differs from Theorem 4.1 in several ways. The most important is that the set where $\operatorname{Re} \phi$ vanishes may extend to the boundary of the region of integration. This precludes the use of the easy deformation $\pi$, because $\mathcal{C}^{\prime \prime}$ is no longer supported on $\{h>\epsilon\}$. Consequently, some work is required to construct a suitable deformation. We do so via notions from stratified Morse theory [GM88].

Tangent vector fields. If $\mathbf{x}$ is a point of the stratum $S$ of the stratified space $\mathcal{M}$, let $T_{\mathbf{x}}(\mathcal{M})$ denote the tangent space to $S$ at $\mathbf{x}$. Because $\mathcal{M}$ is embedded in $\mathbb{C}^{d}$, the tangent spaces may all be identified as subspaces of $\mathbb{C}^{d}$. Thus we have a notion of the tangent bundle $T \mathcal{M}$, a section of which is simply a vector field $f$ on $\mathcal{M} \subseteq \mathbb{C}^{d}$ such that $f(\mathbf{x}) \in T_{\mathbf{x}}(\mathcal{M})$ for all $\mathbf{x}$. A consequence of the two Whitney conditions is the local product structure of a stratified space: a point $\mathbf{p}$ in a $k$ dimensional stratum $S$ of a stratified space $\mathcal{M}$ has a neighborhood in which $\mathcal{M}$ is homeomorphic to some product $S \times X$. According to [GM88], a proof may be found in mimeographed notes of Mather from 1970; it is based on Thom's Isotopy Lemma which takes up fifty pages of the same mimeographed notes. The next lemma is the only place where we use this (or any) consequence of Whitney stratification.

LEMMA 5.1. Let $f$ be a smooth section of the tangent bundle to $S$, that is, $f(s) \in T_{s}(S)$ for $s \in S$. Then each $s \in S$ has a neighborhood in $\mathcal{M}$ on which $f$ may be extended to a smooth section of the tangent bundle.

Proof. Parametrize $\mathcal{M}$ locally by $S \times X$ and extend $f$ by $f(s, x):=f(s)$.

LEMMA 5.2 (vector field near a non-critical point). Let $\mathbf{x}$ be a point of the stratum $S$ of the stratified space $\mathcal{M}$, and suppose $\mathbf{x}$ is not critical for the function $\phi$. Then there is a vector $\mathbf{v} \in T_{\mathbf{x}}(S \otimes \mathcal{M})$ such that $\operatorname{Re}\{\mathrm{d} \phi(\mathbf{v})\}>0$ at $\mathbf{x}$. Furthermore, there is a continuous section $f$ of the tangent bundle in a neighborhood $\mathcal{N}$ of $\mathbf{x}$ such that $\operatorname{Re}\{\mathrm{d} \phi(f(\mathbf{y}))\}>0$ at every $\mathbf{y} \in \mathcal{N}$.

Proof. By non-criticality of $\mathbf{x}$, there is a $\mathbf{w} \in T_{\mathbf{x}}(S)$ with $\mathrm{d} \phi(\mathbf{w})=u \neq 0$ at $\mathbf{x}$. Multiply w componentwise by $\bar{u}$ to obtain $\mathbf{v}$ with $\operatorname{Re}\{\mathrm{d} \phi(\mathbf{v})\}>0$ at $\mathbf{x}$. Use any chart map for $S \otimes \mathbb{C}$ near $\mathbf{x}$ to give a locally trivial coordinatization for the tangent bundle and define a section $f$ to be the constant vector $\mathbf{v}$; then $\operatorname{Re}\{\mathrm{d} \phi(f(\mathbf{y}))\}>0$ on some sufficiently small neighborhood of $\mathbf{x}$ in $S$. Finally, extend to a neighborhood of $\mathbf{x}$ in $\mathcal{M}$ by Lemma 5.1 .

Although we are working in the analytic category, the chains of integration are topological objects, for which we may use $C^{\infty}$ methods (in what follows, even $C^{1}$ methods will do). In particular, a partition of unity argument enhances the local result above to a global result.

LEMMA 5.3 (global vector field, in the absence of critical points). Let $\mathcal{M}$ be a compact stratified space and $\phi$ a smooth function on $\mathcal{M}$ with no critical points. Then there is a global section $f$ of the tangent bundle of $\mathcal{M}$ such that the real part of $\mathrm{d} \phi(f)$ is everywhere positive. 
ProOF. For each point $\mathbf{x} \in \mathcal{M}$, let $f_{\mathbf{x}}$ be a section as in the conclusion of Lemma 5.2, on a neighborhood $U_{\mathbf{x}}$. Cover the compact space $\mathcal{M}$ by finitely many sets $\left\{U_{\mathbf{x}}: \mathbf{x} \in F\right\}$, and let $\left\{\psi_{\mathbf{x}}: \mathbf{x} \in F\right\}$ be a smooth partition of unity subordinate to this finite cover. Define

$$
f(\mathbf{y})=\sum_{\mathbf{x} \in F} \psi_{\mathbf{x}}(\mathbf{y}) f_{\mathbf{x}}(\mathbf{y})
$$

Then $f$ is smooth; it is a section of the tangent bundle because each tangent space is linearly closed; and the real part of $\mathrm{d} \phi(f(\mathbf{y}))$ is positive because we took a convex combination in which each contribution was nonnegative and at least one was positive.

Another partition argument gives our final version of this result.

LEMMA 5.4 (global vector field, vanishing only at critical points). Let $\mathcal{M}$ be a compact stratified space and $\phi$ a smooth function on $\mathcal{M}$ with finitely many critical points. Then there is a global section $f$ of the tangent bundle of $\mathcal{M}$ such that the real part of $\mathrm{d} \phi(f)$ is nonnegative and vanishes only when $\mathbf{y}$ is a critical point.

Proof. Let $\mathcal{M}_{\epsilon}$ be the compact stratified space resulting in the removal of an $\epsilon$-ball around each critical point of $\phi$. Let $g_{\epsilon}$ be a vector field as in the conclusion of Lemma 5.3 with $\mathcal{M}$ replaced by $\mathcal{M}_{\epsilon}$. Let $f_{\epsilon}$ be the product of $g_{\epsilon}$ with a smooth function that is equal to its maximum of 1 on $M_{2 \epsilon}$ and its minimum of 0 on $M_{\epsilon}^{c}$. Let $c_{n}>0$ be chosen small enough so that the magnitudes of all partial derivatives of $c_{n} f_{1 / n}$ of order up to $n$ are at most $2^{-n}$. In the topology of uniform convergence of derivatives of bounded order, the series $\sum_{n} c_{n} f_{n}$ converges to a vector field $f$ with the required properties.

Proof of Theorem 2.3. Let $f$ be a tangent vector field along which $\phi$ increases away from critical points, as given by Lemma 5.4. Such a field gives rise to a differential flow, which, informally, is the solution to $\mathrm{d} \mathbf{p} / \mathrm{d} t=f(\mathbf{p})$. To be more formal, let $\mathbf{x}$ be a point in a stratum $S$ of $\mathcal{M}$. Via a chart map in a neighborhood of $\mathbf{x}$, we solve the ODE $\mathrm{d} \Phi(t) / \mathrm{d} t=f(\Phi(t))$ with initial condition $\Phi(0)=\mathbf{x}$, obtaining a trajectory $\Phi$ on some interval $\left[0, \epsilon_{\mathbf{x}}\right]$ that is supported on $S$. Doing this simultaneously for all $\mathbf{x} \in \mathcal{M}$ results in a map

$$
\Phi: \mathcal{M} \times[0, \epsilon] \rightarrow \mathbb{C}^{d},
$$

with $\Phi(\mathbf{x}, t)$ remaining in $S \otimes \mathbb{C}$ when $\mathbf{x}$ is in the stratum $S$. The map $\Phi$ satisfies $\Phi(\mathbf{x}, 0)=\mathbf{x}$ and $(\mathrm{d} / \mathrm{d} t) \Phi(\mathbf{x}, t)=f(\Phi(\mathbf{x}, t))$. The fact that this may be defined up to time $\epsilon$ for some $\epsilon>0$ is a consequence of the fact that the vector field $f$ is bounded and that a small neighborhood of $\mathcal{M}$ in $\mathcal{M} \otimes \mathbb{C}$ is embedded in $\mathbb{C}^{d}$. Because $f$ is smooth and bounded, for sufficiently small $\epsilon$ the map $\mathbf{x} \mapsto \Phi(\mathbf{x}, \epsilon)$ is a homeomorphism.

The flow reduces the real part of $\phi$ everywhere except at the critical points, which are rest points. Consequently, it defines a homotopy $H(\mathbf{x}, t):=\Phi(\mathbf{x}, \epsilon t)$ between the chain $\mathcal{C}$ representing $\mathcal{M}$ and a chain $\mathcal{C}^{\prime}$ on which the minima of the real part of $\phi$ occur precisely on the set $G$. Recall that $H$ induces a chain homotopy $\mathcal{C}_{H}$ with $\partial \mathcal{C}_{H}=\mathfrak{C}^{\prime}-\mathcal{C}+\partial \mathcal{C} \times \sigma$, where $\sigma$ is a standard 1 -simplex. Let $\omega$ denote the holomorphic $d$-form $A(\mathbf{z}) \exp (-\lambda \phi(\mathbf{z})) \mathrm{d} \mathbf{z}$. Because $\omega$ is a holomorphic $d$-form 
in $\mathbb{C}^{d}$, we have $\mathrm{d} \omega=0$. Now, by Stokes' Theorem,

$$
\begin{aligned}
0 & =\int_{\mathfrak{C}_{H}} \mathrm{~d} \omega \\
& =\int_{\partial \mathfrak{C}_{H}} \omega \\
& =\int_{\mathfrak{C}^{\prime}} \omega-\int_{\mathfrak{C}} \omega-\int_{\partial \mathfrak{C} \times \sigma} \omega .
\end{aligned}
$$

The chain $\partial \mathcal{C} \times \sigma$ is supported on a finite union of spaces $S \otimes \mathcal{C}$, where $S$ is a stratum of dimension at most $d-1$. This chain is supported on a finite union of complex manifolds of dimension at most $d-1$; the integral of a holomorphic $d$-form vanishes over such a chain. Therefore, the last term on the right drops out and we have

$$
\int_{\mathrm{e}} \omega=\int_{\mathrm{e}^{\prime}} \omega
$$

Outside of a neighborhood of $G$ the magnitude of the integrand is exponentially small, so we have shown that there are $d$-chains $\mathcal{C}_{\mathbf{x}}$ supported on arbitrarily small neighborhoods $\mathcal{N}(\mathbf{x})$ of each $\mathbf{x} \in G$ such that

$$
\mathcal{J}(\lambda)-\sum_{\mathbf{x} \in G} \int_{\mathcal{E}_{\mathbf{x}}} \omega
$$

is exponentially small. To finish that proof, we need only show that each $\int_{\mathcal{C}_{\mathbf{x}}} \omega$ has an asymptotic series in decreasing powers of $\lambda$ whose leading term, when $A(\mathbf{x}) \neq 0$, is given by

$$
c_{0}(\mathbf{x})=(2 \pi)^{d / 2} A(\mathbf{x}) e^{\lambda \phi(\mathbf{x})}(\operatorname{det} \mathcal{H}(\mathbf{x}))^{-1 / 2} .
$$

The $d$-chain $\mathcal{C}_{\mathbf{x}}$ may by parametrized by a map $\psi_{\mathbf{x}}: B \rightarrow \mathcal{N}(\mathbf{x})$, mapping the origin to $\mathbf{x}$, where $B$ is the open unit ball in $\mathbb{R}^{d}$. By the chain rule,

$$
\int_{\mathfrak{C}_{\mathbf{x}}} \omega=\int_{B}[A \circ \psi](\mathbf{x}) \exp (-\lambda[\phi \circ \psi(\mathbf{x})]) \operatorname{det} \mathrm{d} \psi(\mathbf{x}) \mathrm{d} \mathbf{x} .
$$

The real part of the analytic phase function $\phi \circ \psi$ has a strict minimum at the origin, so we may apply Theorem 4.1. We obtain an asymptotic expansion whose first term is

$$
\left(\frac{2 \pi}{\lambda}\right)^{d / 2}[A \circ \psi](\mathbf{0}) \operatorname{det} \mathrm{d} \psi(\mathbf{0})\left(\operatorname{det} M_{\mathbf{x}}\right)^{-1 / 2},
$$

where $M_{\mathbf{x}}$ is the Hessian matrix of the function $\phi \circ \psi$. The term $[A \circ \psi](\mathbf{0})$ is equal to $A(\mathbf{x})$. The Hessian matrix of $\phi \circ \psi$ at the origin is given by $M_{\mathbf{x}}=$ $\mathrm{d} \psi(\mathbf{0}) \mathcal{H}(\mathbf{x}) \mathrm{d} \psi(\mathbf{0})$. Thus

$$
\operatorname{det} M_{\mathbf{x}}=(\operatorname{det} \mathrm{d} \psi(\mathbf{0}))^{2} \operatorname{det} \mathcal{H}(\mathbf{x}),
$$

and plugging into (5.3) yields (5.2).

Proof of Corollary 2.4. Lemma 5.4 does not require the critical points to be in the interior, so the argument leading up to (5.1) is still valid. For those points $\mathbf{x}$ in a $(d-1)$-dimensional stratum, use Corollary 2.4 in place of Theorem 4.1 to obtain (5.2) with an extra factor of $1 / 2$. 
REMARK. The reason we do not continue with a litany of special geometries (quarter-spaces, octants, and so forth) is that the case of a half-space is somewhat special. The differential of the change of variables at the origin is a nonsingular map, which must send half-spaces to half-spaces, though it will in general alter angles of any smaller cone.

\section{Examples}

The simplest multidimensional application of our results is a computation from [PW04]. The purpose is to estimate coefficients of a class of bivariate generating functions whose denominator is the product of two smooth divisors. We give only a brief summary of how one arrives at (6.1) from a problem involving generating functions; a complete explanation of this can be found in [PW04, Section 4]. Note, however, that the mathematics of the integral is not contained in that paper, which instead refers to an earlier draft of this one!

Let $v_{1}, v_{2}$ be distinct analytic functions of $z$ with $v_{1}(1)=v_{2}(1)=1,0 \neq v_{1}^{\prime}(1) \neq$ $v_{2}^{\prime}(1) \neq 0$, and such that each $\left|v_{i}(z)\right|$ attains its maximum on $|z|=1$ only at $z=1$. For example, the last condition is satisfied by any pair of aperiodic power series with nonnegative coefficients and radius of convergence greater than 1 .

Consider the generating function $F(z, w)=1 / H(z, w)$, where

$$
H(z, w)=\left(1-w v_{1}(z)\right)\left(1-w v_{2}(z)\right) .
$$

The two branches of the curve $H=0$ intersect only at $(1,1)$, and this intersection is transverse. The Maclaurin coefficients of $F(z, w)=\sum_{r, s} a_{r s} z^{r} w^{s}$ are given by the Cauchy integral formula

$$
a_{r s}=\frac{1}{(2 \pi \mathrm{i})^{2}} \int \frac{\mathrm{d} w \mathrm{~d} z}{z^{r+1} w^{s+1}\left(1-w v_{1}(z)\right)\left(1-w v_{2}(z)\right)},
$$

where the integral is taken over a product of circles centred at $(0,0)$ and of sufficiently small radii.

Pushing the contour out to $|z|=1,|w|=1-\varepsilon$ we obtain the same formula, since $F$ is still analytic inside the product of disks bounded by these latter circles. Pushing the $w$-contour out to $|w|=1+\varepsilon$, using the residue formula on the inner integral and observing that the integral over $|w|=1+\varepsilon$ is exponentially decaying as $s \rightarrow \infty$, we see that

$$
a_{r s} \approx \frac{1}{2 \pi} \int_{|z|=1} \frac{-R_{s}(z)}{z^{r+1}} \mathrm{~d} z
$$

where $\approx$ means that the difference is exponentially decaying as $s \rightarrow \infty$ and $R_{s}(z)$ denotes the sum of residues of $w \mapsto w^{-(s+1)} F(z, w)$ at the roots $w=1 / v_{i}(z)$, $i \in\{1,2\}$.

The residue sum $R_{s}(z)$ can be rewritten in terms of an integral via

$$
-R_{s}(z)=(s+1) \int_{0}^{1}\left[(1-p) v_{1}+p v_{2}\right]^{s} \mathrm{~d} p
$$

and so we have

$$
a_{r s} \approx \frac{s+1}{2 \pi} \int_{|z|=1} z^{-(r+1)} \int_{0}^{1}\left[(1-p) v_{1}(z)+p v_{2}(z)\right]^{s} \mathrm{~d} p \mathrm{~d} z .
$$

In order to cast this into our standard framework, we need to be able to define a branch of the logarithm of $(1-p) v_{1}(z)+p v_{2}(z)$. We do this by localizing on the 
circle $|z|=1$ to a sufficiently small neighbourhood of the point $z=1$. This is possible since the integrand decays exponentially away from $z=1$, by hypotheses on the $v_{i}$, and we shall show that the integral near $z=1$ decays only polynomially.

The substitution $z=e^{\mathrm{i} t}$ converts this to an integral

$$
a_{r s} \approx \frac{s+1}{2 \pi} \int_{\mathcal{N}} \int_{0}^{1} e^{-s \phi(p, t)} A(p, t) \mathrm{d} p \mathrm{~d} t
$$

where $\phi(p, t)=\mathrm{i} r \theta / s+\log \left[(1-p) v_{1}\left(e^{\mathrm{i} t}\right)+p v_{2}\left(e^{\mathrm{i} t}\right)\right], A(p, t)=1$, and $\mathcal{N}$ is a closed interval centred at 0 . To compute asymptotics in the direction $r / s=\kappa$, for fixed $\kappa>0$, we can consider $\phi$ to be independent of $r$ and $s$.

We now asymptotically evaluate (6.1) using Theorem 2.3. We can rewrite the iterated integral as a single integral over the stratified space $\mathcal{M}=\mathcal{N} \times[0,1]$. The phase $\phi$ has nonnegative real part and this fits into our framework. There is a single stationary point, at $(p, z)=(1 / 2,0)$ (note that $\operatorname{Re}\{\phi\}$ is zero for all $(p, 0)$, so Theorem 4.1 does not suffice). This critical point is quadratically nondegenerate, and direct computation using Theorem 2.3 yields

$$
a_{r s}=\frac{1}{\left|v_{1}^{\prime}(1)-v_{2}^{\prime}(1)\right|}+O\left(s^{-1}\right)
$$

when $s \rightarrow \infty$ with $\kappa$ fixed. By keeping track of error terms more explicitly, it is easily shown that this approximation is uniform in $\kappa$ provided $\kappa$ stays in a compact subset of the open interval formed by $v_{1}^{\prime}(1), v_{2}^{\prime}(1)$ (it follows from our assumptions that these numbers are positive real-see [PW04] for more details). This means that $a_{r s}$ is asymptotically constant in any compact subcone of directions away from the boundary formed by lines of inverse slope $\kappa_{i}=v_{i}^{\prime}(1)$.

This example, and in fact a number of cases in [PW04], can also be solved using iterated residues. This is carried out in [BP04]. Iterated residues have the advantage of showing that the $O\left(s^{-1}\right)$ term decays exponentially, but the disadvantage that they do not give any results when $\kappa$ approaches the boundary. The present methods do give boundary results. Corollary 2.4 shows that $a_{r s}$ converges to one-half the right-hand side of $(6.2)$ when $(r, s) \rightarrow \infty$ with $r / s=\kappa_{1}+O(1)$, and a small extension yields a Gaussian limit: letting $\Phi$ denote the standard normal cumulative distribution function, we have

$$
a_{r s}=\frac{\Phi(u)}{\left|v_{1}^{\prime}(1)-v_{2}^{\prime}(1)\right|}+O\left(s^{-1}\right)
$$

when $r, s \rightarrow \infty$ with $\left(r / s-\kappa_{1}\right) / s^{1 / 2} \rightarrow u$.

\section{Further topics}

Higher order terms. We have not emphasized explicit formulae for the higher order terms, giving an equation such as (2.1) only for the leading term in the case where $A(\mathbf{0}) \neq 0$. However, our results establish the validity of existing computations of higher order terms under our more general hypotheses.

To elaborate, we prove Theorem 2.3 by first constructing a change of variables $\mathbf{x} \mapsto \Phi(x, \epsilon)$ homotopic to the identity under which the minimum of $\operatorname{Re}\{\phi\}$ at $\mathbf{0}$ is strict, and then changing variables, again homotopically to the identity, to the standard form. The composition $\psi$ of these two maps is homotopic to the identity 
but is far from explicitly given: while the second map is constructed by an explicitly defined Morse function, the first deformation is the solution to a differential equation and is not particularly explicit.

In [Hör90], Hörmander derives such an explicit formula (assuming smoothness) for integrals of our type where $\mathcal{M}=\mathbb{R}^{d}$ and $A$ has compact support. The formula is indeed rewritten and used in [RW08] to compute higher order terms for generating function applications, in which more restrictive hypotheses preclude the vanishing of $\operatorname{Re}\{\phi\}$ on a curve reaching the boundary of the chain of integration. Their methods, while not covering the cases of interest here, do have the virtue of dealing with the change of variables $\psi$ only through the equation $S=\phi \circ \psi$. In particular, the derivatives of $\psi$ arising in the computation of the new amplitude function $(A \circ \psi) \operatorname{det} \mathrm{d} \psi$ can be computed by implicitly differentiating the equation $S=\phi \circ \psi$. Having found at least one such $\psi$ homotopic to the identity, we are now free to replicate the computations of [RW08] under our more general hypotheses, as follows.

In the case of standard phase, the coefficient of $\lambda^{-(n+d) / 2}$ is given (provided that all $r_{i}$ are even) by

$$
\sum_{|\mathbf{r}|=n} a_{\mathbf{r}} \beta_{\mathbf{r}}
$$

where $a_{\mathbf{r}}$ is the Maclaurin coefficient of $A$ corresponding to the monomial $\mathbf{r}$ and $\beta_{\mathbf{r}}$ is the constant defined in Corollary 3.2. Note that $n$ must be even for this coefficient to be nonzero, so we write $n=2 k$. The differential operator $\partial_{1}^{r_{1}} \cdots \partial_{d}^{r_{d}}$ when applied to $A$ and evaluated at $\mathbf{0}$ yields precisely $\prod_{i} r_{i} ! a_{\mathbf{r}}$. Thus the operator

$$
\sum_{|\mathbf{r}|=k} \frac{\partial_{1}^{2 r_{1}} \cdots \partial_{d}^{2 r_{d}}}{4^{k} r_{1} ! \cdots r_{d} !}
$$

applied to $A$ and evaluated at zero yields the coefficient we seek.

After the Morse lemma is applied using the change of variables $S=\phi \circ \psi$, we need to apply the displayed operator to the new amplitude $(A \circ \psi) \operatorname{det} \mathrm{d} \psi$. The resulting expression evaluated at $\mathbf{x}$ can be computed directly via the rules of Leibniz and Faà di Bruno. Evaluating at $\mathbf{x}$ simplifies some terms, and, as mentioned above, derivatives of $(A \circ \psi) \operatorname{det} \mathrm{d} \psi$ may be computed without explicitly specifying $\psi$.

As a relatively simple example, consider the case $k=1$ and $d=1$. The differential operator reduces to $\frac{1}{4} \partial^{2}$, where $\partial$ denotes differentiation with respect to the variable $x$. Applying this to $(A \circ \psi) \operatorname{det} \mathrm{d} \psi$ yields (with superscripts denoting the order of derivatives and arguments suppressed)

$$
\frac{1}{4}\left(A^{(2)}\left(\psi^{(1)}\right)^{3}+3 A^{(1)} \psi^{(1)} \psi^{(2)}+A^{(0)} \psi^{(3)}\right) .
$$

The defining equation $S=\phi \circ \psi$ can be differentiated to yield the system

$$
\begin{aligned}
2 x & =\phi^{(1)} \psi^{(1)}, \\
2 & =\phi^{(2)}\left[\psi^{(1)}\right]^{2}+\phi^{(1)} \psi^{(2)}, \\
0 & =\phi^{(3)}\left[\psi^{(1)}\right]^{3}+3 \phi^{(2)} \psi^{(1)} \psi^{(2)}+\phi^{(1)} \psi^{(3)}, \\
0 & =\phi^{(4)}\left[\psi^{(1)}\right]^{4}+6 \phi^{(3)}\left[\psi^{(1)}\right]^{2} \psi^{(2)}+4 \phi^{(2)} \psi^{(1)} \psi^{(3)}+3 \phi^{(2)}\left[\psi^{(2)}\right]^{2}+\phi^{(1)} \psi^{(4)} .
\end{aligned}
$$

Evaluating these at the point in question, we see that the terms with highest derivatives of $\psi$ vanish in each equation. The system is triangular and can be solved 
explicitly to obtain

$$
\begin{aligned}
\psi^{(1)} & =\sqrt{\frac{2}{\phi^{(2)}}}, \\
\psi^{(2)} & =\frac{-2 \phi^{(3)}}{3\left[\phi^{(2)}\right]^{2}}, \\
\psi^{(3)} & =\frac{\left[5 \phi^{(3)}\right]^{2}-3 \phi^{(2)} \phi^{(4)}}{3 \sqrt{2}\left[\phi^{(2)}\right]^{7 / 2}} .
\end{aligned}
$$

Putting these together with the expression for the derivative of $(A \circ \psi) \operatorname{det} \mathrm{d} \psi$ above yields an expression for the $\lambda^{-3 / 2}$ term in the integral that is a rational function with denominator $\left[\phi^{(2)}\right]^{7 / 2}$, and with numerator equal to a polynomial in the derivatives of $A$ up to order 2 , and of $\phi$ up to order 4 . In summary, the results of this paper show that the computational apparatus and formulae for higher order terms given in [RW08] hold in the case of complex phase functions integrated over stratified spaces.

Relation to existing literature. As stated at the outset, the aim of the present article has been to outline results that will be useful to the combinatorics community concerning asymptotics of multidimensional integrals with complex phase. Existing treatments may be classified as belonging to one of two types.

Those of the first type are easily accessible but not sufficiently general. These include standard references such as [BH86; Won89], which may be found in engineering libraries as well as mathematics libraries. Also in this class are [Bre94] and [dB81]. These treatments are self-contained, rigorous, and assume little knowledge beyond standard real and complex analysis. These sources treat real phases and purely imaginary phases but do not treat complex phases and do not use inherently complex methods. The treatment by Stein [Ste93], which was written as background for the study of differential operators, is similarly limited (purely imaginary phase, $C^{\infty}$ methods). The book by Paris and Kaminski [PK01] contains great detail on Laplace integrals in dimensions up to three, but has very little on non-real phases. These are the sources typically cited by combinatorialists. When multidimensional contour methods are required, combinatorialists will either reduce somehow to a one-dimensional case, e.g. [BFSS00], or work out the results from scratch, e.g., [Wor04]. Perhaps the closest we have found to an off-the-shelf usable source is [Hör90]. This work is self-contained, considers general complex phases, and is conducive to explicit computation. However, the methods are strictly $C^{\infty}$, and the amplitude function is assumed to be compactly supported in $\mathbb{R}^{d}$.

References of the second type are sufficiently high-powered to do everything we need. However, from our point of view they suffer from a number of drawbacks. Most noticeable is the significant overhead required to make use of these sources. The complex algebraic geometry contained in the work of Pham [Pha85] (extending ideas of [Ler50]) and Malgrange [Mal74], and later [Vas77; Fed77] and the Arnol'd school [Var77; AGZV88], is well beyond the comfort zone of most of the combinatorial community. Furthermore, the results we need, while undeniably implicit in these works, are not always explicitly stated, or easy to find and identify. In some cases, proofs are absent as well [AGZV88; Fed89]. 
It should be noted that the approach taken in these treatments is in some sense the right approach. It is the forerunner to the constructions of [GM88], taking the viewpoint of the Thom-Mather theory. We draw heavily on these ideas in the way we frame and prove our results. The Morse-theoretic deformations we use to prove the lemmas in Section 5 lead, for example, to Pham's "thimbles of Lefschetz." Had we been able to find the exact result we needed in any of these sources, we would have greatly preferred to cite it than to reprove it. However, each of these sources comes with hypotheses that make sense in the context it was written in, but which are too restrictive for our application. These include restrictions on the form of the phase function, the nature of the domain of integration, and specifically the behavior of the integrand on the boundary of the domain. The work of Howls [BH91; DH02] deserves mention because it is more accessible than the other works mentioned in the last two paragraphs. Howls' aim is close to ours: algorithmic understanding of the asymptotics of integrals. These papers emphasize hyperasymptotic theory (asymptotic terms beyond the leading exponential order), which involves non-local classification of the chain of integration and requires added geometric assumptions on the domain of integration.

Acknowledgements. We are indebted to three referees for educating us on the existing literature and for insightful comments on the exposition and the proofs of Theorem 2.3 and Lemma 5.4

\section{References}

[AGZV88] V. I. Arnol'd, S. M. Gusein-Zade, and A. N. Varchenko, Monodromy and Asymptotics of Integrals, vol. II of Singularities of Differentiable Maps, Birkhäuser, Boston, MA, 1988. Translated from the Russian by Hugh Porteous, translation revised by the authors and James Montaldi.

[BBBP08] Y. Baryshnikov, W. Brady, A. Bressler, and R. Pemantle, Two-dimensional quantum random walk, preprint, 34 pp., 2008. Available as arXiv:0810.5495.

[BFSS00] C. Banderier, Ph. Flajolet, G. Schaeffer, and M. Soria, Planar maps and Airy phenomena, pp. 388-402 in Automata, Languages and Programming (Geneva, 2000), no. 1853 in Lecture Notes in Computer Science, Springer-Verlag, Berlin, 2000 .

[BH86] N. Bleistein and R. A. Handelsman, Asymptotic Expansions of Integrals, 2nd edition, Dover, New York, 1986.

[BH91] M. Berry and C. Howls, Hyperasymptotics for integrals with saddles, Proc. Roy. Soc. London Ser. A 434 (1991), 657-675.

[BM93] A. Bertozzi and J. McKenna, Multidimensional residues, generating functions, and their application to queueing networks, SIAM Rev. 35 (1993), 239-268.

[BP04] Y. Baryshnikov and R. Pemantle, Convolutions of inverse linear functions via multivariate residues, preprint, 48 pp., 2004.

[BP08] Y. Baryshnikov and R. Pemantle, Asymptotics of quadratic sequences, III. Quadratic points, preprint, 82 pp., 2008. Available as arXiv:0810.4898.

[Bre94] Karl Breitung. Asymptotic Approximations for Probability Integrals, vol. 1592 of Lecture Notes in Mathematics, Springer-Verlag, Berlin, 1994.

[dB81] N. de Bruijn, Asymptotic Methods in Analysis, 3rd (corrected reprint) edition, Dover, New York, 1981.

[DH02] E. Delabaere and C. Howls, Global asymptotics for multiple integrals with boundaries, Duke Math. J. 112 (2002), 199-264.

[Fed77] M. V. Fedoryuk, Saddle Point Method (in Russian), Nauka, Moscow, 1977. 
[Fed89] M. V. Fedoryuk, Asymptotic methods in analysis, pp. 83-191 in Integral Representations and Asymptotic Methods, vol. 13 of The Encyclopedia of Mathematical Sciences, Springer-Verlag, Berlin, 1989.

[GM88] M. Goresky and R. MacPherson, Stratified Morse Theory, no. 14 of Ergebnisse der Mathematik und ihrer Grenzgebiete (3), Springer-Verlag, Berlin, 1988.

[Hör90] Lars Hörmander. An Introduction to Complex Analysis in Several Variables, 3rd edition, North-Holland, Amsterdam, 1990.

[Ler50] J. Leray. Le calcul différentiel et intégral sur un variété analytique complexe, Bull. Soc. Math. France 87 (1950), 81-180.

[Mal74] B. Malgrange, Intégrales asymptotiques et monodromie, Ann. Sci. École Norm. Sup. (4) 7 (1974), 405-430.

[Pha85] Fr. Pham, La descente des cols par les onglets de Lefschetz, avec vues sur Gauss-Manin, Asterisque 130 (1985), 11-47.

[PK01] R. Paris and D. Kaminski, Asymptotics and Mellin-Barnes Integrals, vol. 85 of The Encyclopedia of Mathematics and its Applications, Cambridge Univ. Press, Cambridge, UK, 2001.

[PW02] R. Pemantle and M. C. Wilson, Asymptotics of multivariate sequences, I. Smooth points of the singular variety, J. Combin. Theory Ser. A 97 (2002), 129-161.

[PW04] R. Pemantle and M. C. Wilson, Asymptotics of multivariate sequences, II. Multiple points of the singular variety, Combin. Probab. Comput. 13 (2004), 735-761.

[PW08] R. Pemantle and M. C. Wilson, Twenty combinatorial examples of asymptotics derived from multivariate generating functions, SIAM Rev. 50 (2008), 199-272.

[RW08] A. Raichev and M.C. Wilson, Asymptotics of coefficients of multivariate generating functions: Improvements for smooth points, Electron. J. Combin. 15 (2008), no. 1, research paper 89, 17 pp.

[Ste93] Elias M. Stein, Harmonic Analysis: Real-Variable Methods, Orthogonality, and Oscillatory Integrals, vol. III in Monographs in Harmonic Analysis, Princeton Univ. Press, Princeton, NJ, 1993. With the assistance of Timothy S. Murphy.

[Var77] A. N. Varchenko. Newton polyhedra and estimation of oscillating integrals, Funct. Anal. Appl. 10 (1977), 175-196.

[Vas77] V. Vasiliev. Asymptotic exponential integrals, Newton's diagram, and the classification of minimal points, Funct. Anal. Appl. 11 (1977), 163-172.

[Wat01] S. Watanabe. Algebraic analysis for nonidentifiable learning machines, Neural Computation 13 (2001), 899-933.

[Won89] R. Wong, Asymptotic Approximations of Integrals, Academic, Boston, MA, 1989.

[Wor04] N. Wormald, Tournaments with many Hamilton cycles, preprint, 2004.

Department of Mathematics, University of Pennsylvania, 209 S. 33rd Street, PhiladelPHIA, PA 19104, USA

E-mail address: pemantle@math.upenn.edu

$U R L$ : www.math. upenn. edu/ ${ }^{\sim}$ pemantle

Department of Computer Science, University of Auckland, Private Bag 92019, Auckland, New ZeAland

E-mail address: mcw@cs.auckland.ac.nz

$U R L:$ http://www.cs.auckland.ac.nz/ mcw/blog 